\title{
Atherosclerosis of the aorta is common in patients with severe aortic stenosis: An intraoperative transesophageal echocardiographic study
}

\author{
Daniel Weisenberg, MD, ${ }^{a}$ Yael Sahar, MS, ${ }^{c}$ Gideon Sahar, MD, ${ }^{b}$ Yaron Shapira, MD, ${ }^{\text {a }}$ Zaza lakobishvili, MD, ${ }^{a}$
} Bernardo A. Vidne, MD, ${ }^{b}$ and Alex Sagie, MD $^{\mathrm{a}}$

From the Dan Sheingarten Echocardiograpy Unit and Valvular Clinic, Department of Cardiology, ${ }^{a}$ and the Department of Cardiothoracic Surgery, ${ }^{\mathrm{b}}$ Rabin Medical Center, Beilinson Campus, Petah Tiqva, affiliated with the Sackler Faculty of Medici$\mathrm{ne}^{\mathrm{c}}$, Tel-Aviv University, Tel-Aviv, Israel.

Received for publication Sept 16, 2004; revisions received Nov 4, 2004; accepted for publication Nov 24, 2004

Address for reprints: Daniel Weisenberg, MD, Echocardiography and Valvular Clinic, The Department of Cardiology, Rabin Medical Center, Beilinson Campus, Petah-Tiqva, 49100, Israel (E-mail: wisnberg@zahav.net.il).

J Thorac Cardiovasc Surg 2005;130:29-32

$0022-5223 / \$ 30.00$

Copyright (C) 2005 by The American Association for Thoracic Surgery

doi:10.1016/j.jtcvs.2004.11.040
Objective: Several studies have recently reported an association between aortic valve calcification and atherosclerosis of the cardiovascular system, suggesting that aortic valve calcification might represent an atherosclerosis-like process. Hence the aim of the present study was to determine whether there is a similar association between aortic stenosis and aortic atheromas.

Methods: We evaluated the records and echocardiographic videotapes of 91 consecutive patients with severe aortic stenosis who underwent intraoperative transesophageal echocardiography before aortic valve replacement to measure the presence and characteristics of aortic atheromas. There were 50 men $(55 \%)$ and 41 women $(45 \%)$. The mean age was $71.9 \pm 9.4$ years (range, $34-91$ years). These patients were compared with 91 sex-and age-matched patients without aortic stenosis who underwent transesophageal echocardiography for various indications. Aortic atheroma was defined as localized intimal thickening of $3 \mathrm{~mm}$ or larger. A lesion was considered complex if there was a plaque extending $5 \mathrm{~mm}$ or more into the aortic lumen; if the lesion was protruding, mobile, or ulcerated; or both.

Results: The aortic stenosis group had significantly higher rates of aortic atheromas $(85 \%$ vs $37 \%, P<.001)$ and complex atheromas $(47 \%$ vs $9 \%, P<.001)$ compared with the control group. In the vast majority of patients in the aortic stenosis group, the aortic atheromas were localized in the aortic arch $(60$ [66\%] patients, with 50\% being complex aortic atheromas) and in the descending aorta (70 [77\%] patients, with $45.7 \%$ being complex aortic atheromas); in only 4 (4.4\%) patients, the aortic atheromas were localized in the ascending aorta (50\% complex aortic atheromas).

Conclusions: There is a strong association between the presence of severe aortic stenosis and the presence and severity of aortic atheromas, suggesting that aortic stenosis might be a manifestation of the atherosclerotic process. These findings imply that (1) aggressive atherosclerotic risk-factor modification for patients with aortic stenosis might be advisable and (2) consideration of evaluation of the aorta by means of transesophageal echocardiography before aortic valve replacement in selected patients might be helpful.

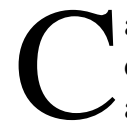

alcific aortic stenosis (AS) has become the most common cardiac valvular disease in developed countries, affecting $2 \%$ to $3 \%$ of the population by the age of 65 years. ${ }^{1,2}$ In the recently published European Heart Survey on valvular disease comprising 5001 patients, AS was the most frequent, single, native, left-sided valve disease, with $82 \%$ of the cases being of "degenerative" cause. ${ }^{1}$

The early lesions of degenerative AS have some similarities to arterial atherosclerosis plaques. ${ }^{3}$ Several studies have recently reported an association between aortic valve calcification and atherosclerosis of the cardiovascular system, suggesting that aortic valve calcification might represent an atherosclerosis-like process. ${ }^{4,5}$ 
TABLE 1. Clinical characteristics and prevalence of aortic atheromas in the AS group and control subjects

\begin{tabular}{lccc}
\hline Characteristic & $\begin{array}{c}\text { AS group } \\
(\mathbf{n}=\mathbf{9 1 )}\end{array}$ & $\begin{array}{c}\text { Control } \\
\text { group } \\
(\mathbf{n}=\mathbf{9 1})\end{array}$ & $\begin{array}{c}\boldsymbol{P} \\
\text { value }\end{array}$ \\
\hline Age (y) & $71.9 \pm 9.4$ & $70.4 \pm 8.4$ & NS \\
Male sex (\%) & 55 & 59 & NS \\
Hypertension (\%) & 48 & 44 & NS \\
Hypercholesterolemia (\%) & 29.7 & 31.9 & NS \\
Diabetes (\%) & 18.7 & 16.5 & NS \\
Aortic atheromas ( $\geq 3 \mathrm{~mm})$ & $77(85 \%)$ & $34(37 \%)$ & $<.001$ \\
Complex atheromas & $43(47 \%)$ & $8(9 \%)$ & $<.001$ \\
\hline
\end{tabular}

$A S$, Aortic stenosis; NS, not significant.

Furthermore, recent studies have demonstrated an association between atherosclerotic risk factors and aortic valve disease. $^{6,7}$

Hence the aim of the present study was to determine whether there is a similar association between severe AS and atherosclerosis of the thoracic aorta imaged by means of transesophageal echocardiography (TEE).

\section{Methods \\ Study Group}

The study population was composed of 91 consecutive patients with severe AS who underwent intraoperative TEE before aortic valve replacement between January 1, 2002, and July 31, 2003, in a single tertiary medical center. There were 50 men (55\%) and 41 women $(45 \%)$. The mean age was $71.9 \pm 9.4$ years (range, 34-91 years). These patients were compared with 91 sex- and agematched patients (59 men and 32 women; mean age, $70.4 \pm 8.4$ years; range, 44-90 years) without AS who underwent TEE for various indications during the same period (control group). Indications for TEE referral in the control group were (1) evaluation for suspected cardiac source of thromboembolism (25.8\%), (2) suspected infective endocarditis (23.5\%), (3) evaluation of nativeprosthetic valve $(22.3 \%)$, (4) before cardioversion of atrial fibrillation (11.7\%), and (5) "other" (16.4\%).

The atherosclerotic risk factors considered in this study were diabetes mellitus, hypertension, and hypercholesterolemia. Diabetes was defined as hyperglycemia requiring pharmacologic therapy. Hypertension was defined as either a systolic or diastolic increase in blood pressure $(>140 / 90 \mathrm{~mm} \mathrm{Hg}$ ) or ongoing antihypertensive pharmacologic therapy. Hypercholesterolemia was defined as a total cholesterol level of greater than $200 \mathrm{mg} / \mathrm{dL}$.

\section{Echocardiographic Studies}

All intraoperative TEE studies were performed by cardiologists experienced in intraoperative TEE (DW, YS, AS) using a 3.7/5- or 4/7-MHz multiplane transducer of Phillips (Andover, Mass) or 6T General Electric Vingmed Ultrasound (Harten, Norway). The ultrasonographs used were Agilent 5500 or Vivid 3 Premium digital cardiovascular ultrasound scanners. Each study was recorded on super-VHS tape. Severe AS was defined as a reduction in valve area to less than $1 \mathrm{~cm}^{2}$ according to the American College of
TABLE 2. Prevalence of aortic atheromas in the AS group according to the main causes of the disease

\begin{tabular}{lccc}
\hline & $\begin{array}{c}\text { Degenerative } \\
\text { AS } \\
(\mathbf{n}=\mathbf{5 9})\end{array}$ & $\begin{array}{c}\text { AS of other } \\
\text { causes } \\
(\mathbf{n}=\mathbf{3 2})\end{array}$ & $\begin{array}{c}\boldsymbol{P} \\
\text { value }\end{array}$ \\
\hline Aortic atheromas $(\geq 3 \mathrm{~mm})$ & $53(89.9 \%)$ & $25(78.1 \%)$ & $\mathrm{NS}$ \\
Complex atheromas & $35(59.3 \%)$ & $8(25.0 \%)$ & .004 \\
\hline
\end{tabular}

$A S$, Aortic stenosis; NS, not significant.

Cardiology/American Heart Association guidelines ${ }^{8}$ or mean gradient of greater than $50 \mathrm{~mm} \mathrm{Hg}$ across the aortic valve.

After complete cardiac examination, aortic images were obtained by rotating the transducer posteriorly and advancing it to the distal esophagus. Then it was slowly withdrawn to obtain images from the distal thoracic aorta to the aortic arch. The ascending aorta was imaged in the midesophageal window at the level of the long axis of the aortic valve $\left(120^{\circ}-160^{\circ}\right)$. The aortic intima was evaluated for changes in thickening, protrusion, mobile components, or ulceration. Aortic atheroma (AA) was defined as localized intimal thickening of $3 \mathrm{~mm}$ or greater. A lesion was considered complex according to the presence of 1 or more of the following: (1) thickening of the intima extending $5 \mathrm{~mm}$ or more into the aortic lumen, (2) disruption or irregularities of the intimal surface (ulceration), (3) mobile component of the atheroma, and (4) protruding atheroma.

\section{Statistical Analysis}

Numeric values are reported as means \pm SD or as a proportion of the sample size. Comparisons between the study and control groups were made with $\chi^{2}$ analysis for categorical data and the Student $t$ test for continuous data.

\section{Results}

The clinical characteristics and prevalence of AAs in the 2 groups are shown in Table 1. No differences were found between the groups regarding risk factors for atherosclerosis. The cause of AS was degenerative in 59 (64.8\%) patients, rheumatic in $14(15.3 \%)$ patients, bicuspid in 6 $(6.6 \%)$ patients, and undetermined in $12(13.1 \%)$ patients.

Table 2 shows the prevalence of AAs in the AS group according to the cause of the disease. The AS group had significantly higher rates of AAs $(85 \%$ vs $37 \%, P<.001)$ and complex atheromas (47\% vs $9 \%, P<.001)$ compared with the control group. In the AS group the vast majority of AAs were localized in the aortic arch (60 [66\%] patients, with $50 \%$ being complex atheromas) and in the descending aorta (70 [77\%] patients, with $45 \%$ being complex atheromas); in only $4(4.4 \%)$ patients, the AAs were localized in the ascending aorta (50\% complex atheromas). Forty-four (48.3\%) patients in the AS group underwent concomitant coronary artery bypass grafting, and in 5 of them it was a redo procedure. Four (4.4\%) patients experienced a perioperative stroke, and in 3 of them complex atheromas in the 
aortic arch were present on TEE. The operative mortality was $1.1 \%$.

\section{Discussion}

To the best of our knowledge, this is the first clinical report showing a strong and significant association between the presence of severe AS and AAs on echocardiography. Our findings further support the hypothesis of AS being the result of an atherosclerotic-like process. These data might have important implications in the clinical management of this common disease. Calcific AS is a progressive valvular disease with a high prevalence and, despite the excellent outcome of aortic valve replacement, ${ }^{1,9}$ still remains a significant cause of morbidity and mortality.

Once thought to be a degenerative and age-related condition, several studies in the recent years have shown convincing evidence that calcific AS is the consequence of an active inflammatory process with some similarities to atherosclerosis. ${ }^{2,10}$

Otto and colleagues ${ }^{3}$ have shown that the early lesion of degenerative AS is an active inflammatory process characterized by means of lipid deposition, macrophages, and T-cell infiltration similar to that seen in the atherosclerotic plaque. Moreover, risk factors for arterial atherosclerosis (ie, male sex, tobacco use, hypercholesterolemia, hypertension, and diabetes mellitus) have all been associated with the development and more rapid progression of AS. ${ }^{7,11-13}$ The combination of clinical findings and experimental observations support the hypothesis that calcific degenerative AS is the result of an active inflammatory cellular process, an atherosclerosis-like process involving the aortic valve.

Our group was the first to report a strong and significant association between the presence of aortic valve calcification and AAs in a group of 96 patients who underwent TEE for different causes. Simple atheromas ( $\geq 3 \mathrm{~mm}$ ) were found in $86 \%$ versus $30 \%$ and complex atheromas in $69 \%$ versus $18 \%$ in the aortic valve calcification group compared with the control subjects. ${ }^{5}$

Agmon and associates, ${ }^{14}$ in a population-based study of 381 subjects, demonstrated that multiple risk factors (age, male sex, hypertension, obesity, and homocystein levels) and aortic atherosclerosis evidenced by means of echocardiography of the aorta were associated with aortic valve sclerosis.

Several retrospective studies have suggested that aggressive treatment with lipid-lowering agents (statins) might slow the progression of valvular calcification. ${ }^{15-17}$ Novaro and coworkers, ${ }^{15}$ in a retrospective study of 174 patients with mild-to-moderate calcific AS, showed that statin use was a significant independent predictor of a smaller decrease in valve area and a lesser increase in peak gradient during a mean follow-up of 21 months. Shavelle and associates, ${ }^{17}$ in a retrospective analysis of 65 patients with calcific AS, showed that statin treatment was associated with a $63 \%$ lower rate of aortic valve calcium accumulation, as measured by means of electron-beam computed tomography. Future prospective randomized trials will be required to establish the potential benefit of these agents in slowing the progression of AS and whether it might be helpful in the advanced stage of this disease.

Aortic valve replacement is still the mainstay of therapy for symptomatic severe AS. The operative mortality for AS has dramatically decreased in recent years, being around $3.1 \%$ nowadays. ${ }^{1}$ However, the operative risk might be higher in the elderly and in the presence of comorbidities, such as coronary artery disease, renal disease, and cerebrovascular disease. Embolic stroke occurs in $2 \%$ to $7 \%$ of patients during cardiac surgery involving cardiopulmonary bypass, especially in the elderly. ${ }^{18,19}$ Furthermore, in one series patients with aortic arch atheromas had a significantly higher incidence of perioperative stroke than did those without atheromas (15\% vs $2 \%){ }^{20}$ In another study of 268 patients who underwent TEE, the presence of aortic arch atheromas of $5 \mathrm{~mm}$ or larger increased the perioperative stroke rate by 6 times and more than doubled the in-hospital mortality after heart surgery. ${ }^{21}$ The incidence of perioperative stroke in our study (4 [4.4\%] patients) is in accordance with that found in the literature; however, it is remarkable that complex aortic arch atheromas were found in 3 of 4 of these patients, raising the suspicion of a cause-event link.

Our findings might have important practical implications in the management of patients with severe AS. Taking into account that more than half of the patients with severe AS who are referred to surgical intervention are more than 70 years old and that a third of them require concomitant coronary bypass grafting (a factor that almost doubles the operative mortality), ${ }^{1}$ we suggest that the presence of AAs should be taken into account when analyzing the risk/benefit ratio for these patients when aortic valve replacement is contemplated. We consider TEE to be the best tool for evaluating the presence and complexity of AAs. Therefore we suggest that TEE might be performed as part of the preoperative evaluation of a selected group of patients with severe AS who do not have strong indications for aortic valve replacement, such as elderly patients with no or equivocal symptoms and with mild findings during exercise testing or patients with concomitant comorbidities. This might provide patients and surgeons with important information regarding the presence of AAs that might expose patients to a higher risk of stroke and perioperative mortality.

In conclusion, there is a strong association between the presence of severe AS and the presence and severity of AAs, suggesting that AS might be a manifestation of the atherosclerotic process. These findings imply that (1) aggressive atherosclerotic risk-factor modification for patients with AS might be advisable and (2) consideration of eval- 
uation of the aorta by means of TEE before aortic valve replacement in selected patients might be helpful.

\section{References}

1. Iung B, Baron G, Butchart E, et al. A prospective survey of patients with valvular heart disease in Europe: the Euro Heart Survey on Valvular Heart Disease. Eur Heart J. 2003;24:1231-43.

2. Lindroos M, Kupari M, Heikkila J, Tilvis R. Prevalence of aortic valve abnormalities in the elderly: an echocardiographic study of a random population sample. J Am Coll Cardiol. 1993;21:1220-5.

3. Otto CM, Kuusisto J, Reinchenbach DD, Gown AM, O'Brien KD. Characterization of the early lesion of "degenerative" valvular aortic stenosis: histologic and inmunohistochemical studies. Circulation. 1994;90:844-53.

4. Otto CM, Lind BK, Kitzman DW, Gersh BJ, Siscovick DS. Association of aortic valve sclerosis with cardiovascular mortality and morbidity in the elderly. $N$ Engl J Med. 1999;341:142-7.

5. Adler Y, Vaturi M, Wiser I, et al. Nonobstructive aortic valve calcium as a window to atherosclerosis of the aorta. Am J Cardiol. 2000;86: 68-71.

6. Lindroos M, Kupari M, Valvanne J, Strandberg T, Heikkila J, Tilvis R. Factors associated with calcific aortic valve degeneration in the elderly. Eur Heart J. 1994;15:865-70.

7. Stewart BF, Siscovick D, Lind BK, et al. Clinical factors associated with calcific aortic valve disease. J Am Coll Cardiol. 1997;29:630-4.

8. Bonow RO, Carabello B, De Leon AC, et al. ACC/AHA Guidelines for the management of patients with valvular heart disease. J Am Coll Cardiol. 1998;32:1486-588.

9. Lindblom D, Lindblom U, Qvist J, Lundstrom H. Long-term relative survival rate after heart valve replacement. J Am Coll Cardiol. 1990; 15:566-73.

10. O' Brien KD, Reichenbach DD, Marcovina SM, Kuusisto J, Alpers CE, Otto CM. Apolipoproteins B, (a), and E accumulate in the morphologically early lesion of "degenerative" valvular aortic stenosis. Arterioscler Thromb Vasc Biol. 1996;16:523-32.
11. Palta S, Pai AM, Gill KS, Pai RJ. New insights into the progression of aortic stenosis: implications for secondary prevention. Circulation. 2000;101:2497-502

12. Peter M, Hoffmann A, Parker C, Luscher T, Burckhardt D. Progression of aortic stenosis: role of age and concomitant coronary artery disease. Chest. 1993;103:1715-9.

13. Aronow W, Chul A, Kronzon I, Goldman M. Association of coronary risk factors and use of statins with progression of mild valvular aortic stenosis in older persons. Am J Cardiol. 2001;88:693-5.

14. Agmon Y, Khandheria B, Meissner I, et al. Aortic valve sclerosis and aortic atherosclerosis: different manifestations of the same disease? J Am Coll Cardiol. 2001;38:827-34.

15. Novaro GM, Tiong TY, Pearce GL, Lauer M, Sprecher D, Griffin B. Effect of hydroxymethylglutaryl coenzyme A reductase inhibitors on the progression of calcific aortic stenosis. Circulation. 2001;104: 2205-9.

16. Bellamy MF, Pellika PA, Klarich KW, Tajik AJ, Enriquez-Sarano M. Association of cholesterol levels, hydroxymethylglutaryl coenzyme-A reductase treatment, and progression of aortic stenosis in the community. J Am Coll Cardiol. 2002;40:1723-30.

17. Shavelle DM, Takasu J, Budoff MJ, Mao S, Zhao XQ, O'Brien KD. HMG CoA reductase inhibitor (statin) and aortic valve calcium. Lancet. 2002;359:1125-6.

18. Gardner TJ, Horneffer P, Manolio TA, et al. Stroke following coronary artery bypass grafting: a ten year study. Ann Thorac Surg. 1985;40: 574-81.

19. Salomon NW, Page US, Bigelow JC, Krause AH, Okies JE, Metzdorff MT. Coronary artery bypass grafting in elderly patients. Comparative results in a consecutive series of 469 patients older than 75 years. J Thorac Cardiovasc Surg. 1991;101:209-18.

20. Katz ES, Tunick PA, Rusinek H, Ribakove G, Spencer FC, Kronzon I. Protruding aortic atheromas predict stroke in elderly patients undergoing cardiopulmonary bypass: a review of our experience with intraoperative transesophageal echocardiography. J Am Coll Cardiol. 1992; 20:70-7.

21. Stern A, Tunick PA, Culliford AT, et al. Protruding aortic arch atheromas: risk of stroke during heart surgery with and without aortic arch endarterectomy. Am Heart J. 1999;138:746-52 